\title{
Reflets
}

Revue d'intervention sociale et communautaire

\section{Dépasser une double invalidation : la lutte contre l'exclusion sociale de jeunes femmes immigrantes et de leur communauté}

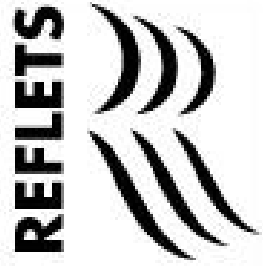

\section{Marie Drolet et Hindia Mohamoud}

Volume 16, numéro 2, 2010

L'intervention sociale en contextes minoritaires : penser la complexité et la multiplicité des processus de minorisation

URI : https://id.erudit.org/iderudit/1000315ar

DOI : https://doi.org/10.7202/1000315ar

Aller au sommaire du numéro

Éditeur(s)

Reflets, Revue d'intervention sociale et communautaire

ISSN

1203-4576 (imprimé)

1712-8498 (numérique)

Découvrir la revue

Citer cet article

Drolet, M. \& Mohamoud, H. (2010). Dépasser une double invalidation : la lutte contre l'exclusion sociale de jeunes femmes immigrantes et de leur communauté. Reflets, 16(2), 90-117. https://doi.org/10.7202/1000315ar

\section{Résumé de l'article}

L'approche théorique en émergence d'Essed (1991; 2002) sur l'exclusion ethnoculturelle ouvre une voie prometteuse pour mieux comprendre l'actualisation de ce concept. Fondée sur la prémisse que le racisme est inhérent à l'ordre social et à la culture dominante, l'exclusion est expérimentée à travers des pratiques familières, systématiques et récurrentes, cumulées au fil du temps.

Au cours de l'hiver 2006, le Conseil de planification sociale d'Ottawa (CSPO) a organisé douze groupes d'entretien regroupant 64 volontaires, et ce, dans le but de cerner les pratiques d'exclusion qui touchent des familles d'origine somalienne, chinoise et libanaise. L'échantillon typique respecte le scénario migratoire et la diversité de chacune des trois communautés. Il se divise en quatre groupes : un de jeunes femmes, un de jeunes hommes, un de mères et un de pères.

Les jeunes femmes de notre échantillon affrontent des barrières à leur intégration à la société canadienne, barrières érigées autant par leur famille et leur communauté d'origine que par des membres de la culture dominante. Leurs communautés étant préoccupées par d'autres enjeux, souvent socio-économiques, les jeunes femmes expérimentent donc une invalidation de leurs perceptions et un sentiment d'être différentes de leur culture d'origine. Les stratégies qu'elles adoptent pour s’y opposer reçoivent peu d'échos; ce silence augmente l'invalidation ressentie.
Tous droits réservés @ Reflets, Revue d’intervention sociale et communautaire, 2010
Ce document est protégé par la loi sur le droit d'auteur. L'utilisation des services d'Érudit (y compris la reproduction) est assujettie à sa politique d'utilisation que vous pouvez consulter en ligne. 


\section{Dépasser une double invalidation : la lutte contre l'exclusion sociale de jeunes femmes immigrantes et de leur communauté ${ }^{1}$}

Marie Drolet, Ph. D., t.s.

Professeure agrégée, École de service social, Université d'Ottawa

Hindia Mohamoud

$M A$ science économique,

Directrice du groupe communautaire PLIO

(Partenariat local pour l'immigration de la Ville d'Ottawa)

\section{Résumé}

L'approche théorique en émergence d'Essed (1991; 2002) sur l'exclusion ethnoculturelle ouvre une voie prometteuse pour mieux comprendre l'actualisation de ce concept. Fondée sur la prémisse que le racisme est inhérent à l'ordre social et à la culture dominante, l'exclusion est expérimentée à travers des pratiques familières, systématiques et récurrentes, cumulées au fil du temps.

Au cours de l'hiver 2006, le Conseil de planification sociale d'Ottawa (CSPO) a organisé douze groupes d'entretien regroupant 64 volontaires, et ce, dans le but de cerner les pratiques d'exclusion qui touchent des familles d'origine somalienne, chinoise et libanaise. L'échantillon typique respecte le scénario migratoire et la diversité de chacune des trois communautés. Il se divise en quatre groupes : un de jeunes femmes, un de jeunes hommes, un de mères et un de pères. 
Les jeunes femmes de notre échantillon affrontent des barrières à leur intégration à la société canadienne, barrières érigées autant par leur famille et leur communauté d'origine que par des membres de la culture dominante. Leurs communautés étant préoccupées par d'autres enjeux, souvent socio-économiques, les jeunes femmes expérimentent donc une invalidation de leurs perceptions et un sentiment d'être différentes de leur culture d'origine. Les stratégies qu'elles adoptent pour s'y opposer reçoivent peu d'échos; ce silence augmente l'invalidation ressentie.

Mots clés : jeunes femmes immigrantes, invalidation, racisme, socialisation de genre, Essed

\section{Abstract}

Essed's $(1991 ; 2002)$ emerging theoretical approach to ethnocultural exclusion opens up a promising channel towards better understanding of the realization of this concept. Based on the premise that racism is inherent in social order in general and in the dominant culture in particular, exclusion is experienced through systematic and recurring daily experiences that accumulate over time.

During the winter of 2006, the Social Planning Council of Ottawa (SPCOttawa) set up 12 focus groups comprising 64 volunteers with the aim of identifying exclusionary behaviours affecting families of Somali, Chinese and Lebanese origins. A typical sample took into account the migratory background and diversity of all three communities. Each sample was broken down into four sub-groups : young women, young men, mothers, fathers.

The young women in our sample group confront barriers to integration into Canadian society that have been created as much by their families and cultural communities as by members of the dominant culture at large. Since their communities are preoccupied with socioeconomic issues, these young women experience an invalidation of their perceptions by them as well as the feeling of being different from their culture of origin. The strategies they adopt to counter such invalidation are scarcely 
acknowledged; in turn, this wall of silence increases the degree of invalidation felt by these women.

Key words : young immigrant women, invalidation, racism, gender socialization, Essed

\section{Introduction}

"On dénombre maintenant 58520

francophones faisant partie d'une minorité visible. Les trois quarts d'entre eux sont nés à l'extérieur du Canada : un tiers venant d'Afrique, un tiers d'Asie et près d'un cinquième du Moyen-Orient. "
La diversité culturelle et la complexité grandissante qui caractérisent les pays occidentaux, dont le Canada, offrent de nombreux défis à l'intervention sociale. En effet, plus d'un million d'immigrants se sont installés au Canada entre 2001 et 2006; les trois quarts de ces personnes appartiennent à une minorité visible (Statistique Canada, 2008). L'Ontario français connaît aussi une telle transformation. On dénombre maintenant 58520 francophones faisant partie d'une minorité visible. Les trois quarts d'entre eux sont nés à l'extérieur du Canada : un tiers venant d'Afrique, un tiers d'Asie et près d'un cinquième du MoyenOrient. Même si ces francophones ont un niveau de scolarité plus élevé que ceux de la population générale, leur revenu annuel moyen n'en demeure pas moins inférieur de 6000 \$ (Office des affaires francophones, 2005).

À Ottawa où s'est menée l'étude entreprise par le Conseil de planification sociale dont il est ici question, l'immigration s'avère un phénomène assez récent. De fait, presque une personne sur 5 $(18,1 \%)$ de cette région métropolitaine est née à l'étranger. Plus de la moitié de ces personnes immigrantes $(53,1 \%)$ sont arrivées depuis 1991 (Statistique Canada,2007). Ces changements au sein de sa population correspondent aux tendances canadiennes et à celles de l'Ontario français. La transition d'une immigration européenne à des nouveaux arrivants venant très majoritairement d'Afrique, d'Asie et du Moyen-Orient a pour effet que la population de minorité visible correspond maintenant à $20 \%$ de celle d'Ottawa et a augmenté de $63 \%$ en dix ans (Statistique Canada, 2008). La population noire y est la plus nombreuse, correspondant au quart de ces personnes $(24,1 \%)$, suivie de la population d'origine 
"...il importe

de souligner que

l'intégration sociale

et économique des

personnes immigrantes

(parfois même de

deuxième et de

troisième génération)

se réalise de plus en

plus lentement au

Canada. » chinoise qui compte pour le cinquième environ $(18,9 \%)$ des personnes de minorité visible. L'arabe y est la langue maternelle la plus parlée après l'anglais et le français (Rogers etYoung, 2007), les personnes d'origine arabe représentant le deuxième groupe ethnoculturel où la croissance est la plus rapide (18.3\%) depuis 2001 (Greenberg, 2008).

La croissance de la main d'œuvre étant à la source de cette immigration, cette transformation démographique est appelée à se poursuivre. Par ailleurs, il importe de souligner que l'intégration sociale et économique des personnes immigrantes (parfois même de deuxième et de troisième génération) se réalise de plus en plus lentement au Canada (Papillon, 2004; Omidvar et Richmond, 2003, Reitz, 1998) et à Ottawa.

En tenant compte de ces considérations et dans le but de bien cerner la situation des personnes de minorité visible, le Conseil de planification sociale d'Ottawa (CPSO) a mené de 2005 à 2007 une recherche intitulée Communities Within : Diversity and Exclusion in Ottawa (Moscovitch et Mohamoud, 2005; CPSO, 2008). Les expériences d'exclusion et d'inclusion variant d'une communauté ethnoculturelle à l'autre, le Conseil a choisi de documenter la spécificité de trois d'entre elles selon une méthode d'étude de cas fondée sur des entretiens de groupe et des entrevues avec des chefs de file de chacune d'elles (CPSO, 2008). Le terme communauté est ici défini comme un ensemble de relations complexes examinées dans un cadre déterminé où sont présents des processus d'organisation et de participation (Boudon, et collab., 1993). Dans ses choix de communautés, le CPSO a aussi tenu compte de la représentation francophone chez les personnes de minorité visible.

En accord avec les résultats du recensement, le CPSO (2005) a tout d'abord choisi la communauté somalienne pour illustrer l'expérience de la population noire parce qu'elle y est la plus populeuse - arrivée au cours des années 1990, souvent avec le statut de réfugié - et de religion musulmane. La communauté chinoise a été retenue puisqu'elle constituait en 2001 la minorité visible la plus nombreuse au Canada. Précisons cependant que cette croissance s'est accélérée avec l'immigration récente. En 
"...l'analyse du CPSO (2008) met en lumière l'exclusion économique que vivent ces trois communautés de même que la «racialisation » de la pauvreté..." effet, malgré la longue tradition de cette communauté au pays, seulement une personne sur quatre d'origine chinoise est née au Canada. (Statistique Canada, 2008). Quant à la représentation arabe, le CPSO (2005) a porté son choix sur la communauté libanaise parce qu'elle est la plus importante à Ottawa, et de dénomination chrétienne ou musulmane. À ce titre, Hayani (1999) avance qu'au Canada, de 1946 à 1997, près du tiers (30,9\%) de l'immigration arabe venait du Liban et que $60 \%$ de ces personnes étaient d'ascendance chrétienne. Outre cette immigration ancienne, la situation politique récente du Liban continue d'alimenter le flux migratoire. Une personne sur quatre de la minorité arabe canadienne et née à l'étranger est libanaise (Statistique Canada, 2008). Les communautés somalienne et libanaise comptent des membres francophones.

Les données émanant de l'étude du CPSO ont fait l'objet de l'analyse secondaire présentée dans cet article.Tandis que l'analyse du CPSO (2008) met en lumière l'exclusion économique que vivent ces trois communautés de même que la "racialisation » de la pauvreté, cet article met l'accent sur l'exclusion sociale vécue au quotidien, plus spécialement par les jeunes femmes issues de ces trois collectivités. Tout d'abord, seront établis un bilan de l'approche théorique d'Essed $(1991 ; 2002)$ sur l'exclusion ethnoculturelle perceptible au quotidien et une brève synthèse des principaux écrits sur la socialisation de genre appliquée aux analyses interculturelles. Puis, les enjeux d'exclusion sociale et de socialisation de genre seront discutés pour ces communautés, en mettant un accent sur le quotidien des jeunes femmes de chacun de ces groupes. Cette analyse exploratoire sera réalisée à partir de douze groupes d'entretien $(\mathrm{N}=64)$ qui se répartissent ainsi pour chaque collectivité : un groupe de jeunes femmes, un de jeunes hommes, un de mères et un de pères. Nous terminerons avec des pistes de réflexion que ce cadre d'analyse sur l'exclusion ethnoculturelle vécue au quotidien entraîne sur la pratique du service social. 


\section{Des jeunes femmes au quotidien complexe}

Pour bien mettre en lumière le vécu complexe des jeunes femmes de trois minorités visibles au Canada, nous avons choisi d'explorer leurs propos à l'aide de deux cadres théoriques reconnus et complémentaires, soit l'exclusion ethnoculturelle quotidienne telle que formulée par Essed et la socialisation de genre appliquée aux analyses interculturelles. En ce qui a trait à la théorie de l'exclusion quotidienne, Essed l'ayant développée à partir de multiples récits de femmes de la communauté noire venant de diverses origines, cette assise empirique en confirme la pertinence aux fins de cette analyse.

\section{L'exclusion ethnoculturelle associée au racisme}

L'approche théorique d'Essed (1991; 2002) sur l'exclusion ethnoculturelle ouvre une voie prometteuse pour mieux saisir

"L'exclusion

sociale, ici appliquée

aux dimensions

ethnoculturelles, est

considérée à la fois

comme un résultat et

un processus. » ce concept au sein de la réalité quotidienne. L'exclusion sociale, ici appliquée aux dimensions ethnoculturelles, est considérée à la fois comme un résultat et un processus (Cushing, 2003). En tant que résultat, cette exclusion correspond à diverses privations sur le plan matériel, politique et relationnel. Sen (2000) précise que l'exclusion sociale se distingue de la pauvreté en raison des composantes relationnelles de ce concept. Tout en incluant des privations économiques, de même que des difficultés d'accès à se réaliser et à vivre le quotidien — vraiment par choix? l'exclusion sociale attire l'attention sur la dimension relationnelle, comme une voie vers des ressources et ainsi, vers des résultats désirés.

En guise de processus, l'exclusion sociale est vue comme un ensemble complexe, multidimensionnel et dynamique de mécanismes qui activent des structures sociales d'iniquité et que subissent tous les jours les personnes exclues (Cushing, 2003; Saloojee, 2003). Autant vivre en contexte de pauvreté provoque à la longue de plus en plus de pauvreté et un sentiment d'impuissance, autant être en contexte d'exclusion appauvrit le réseau social et amène à perdre ses propres capacités d'action et de 
"Le racisme y est défini comme une hiérarchisation de valeur ou de pouvoir entre les groupes ou les individus. ")

"...l'exclusion
ethnoculturelle est
expérimentée à travers
des pratiques familières,
systématiques et
récurrentes, cumulées
au fil du temps
et intégrées aux
routines de la vie
quotidienne. "

réaction (DeHaan, 1999). Cependant, Saloojee (2003) maintient que le concept d'exclusion sociale inclut les notions de division de classes sociales, les inégalités de genre, de même que le racisme. Il affirme également que ces formes d'iniquité demandent que l'on porte une attention spéciale à chacune d'elles. C'est dans ce sens que les travaux d'Essed constituent un regard particulier sur l'exclusion des femmes de minorités visibles, doublement exclues en raison du racisme et du sexisme.

Essed a construit son approche théorique de l'exclusion ethnoculturelle quotidienne sur la prémisse que le racisme est partie inhérente de l'ordre social et de la culture dominante. Le racisme y est défini comme une hiérarchisation de valeur ou de pouvoir entre les groupes ou les individus. Cette hiérarchie se fonde sur trois aspects présents dans le groupe majoritaire :

1) des perceptions et croyances communes à propos des personnes exclues;

2) un partage d'intérêts contre ces dernières ou la distinction entre le "nous » et « eux »;

3) un parcours historique qui façonne des relations inégales de pouvoir.

Cette auteure dépasse une compréhension dichotomique du phénomène, soit d'une part, la discrimination systémique ou structurelle et d'autre part, des gestes touchant directement des individus, et alors perçus comme des événements isolés, anecdotiques ou anodins.

Selon Essed, l'exclusion ethnoculturelle est expérimentée à travers des pratiques familières, systématiques et récurrentes, cumulées au fil du temps et intégrées aux routines de la vie quotidienne. Les personnes subissant cette exclusion l'observent et l'analysent à partir de leur propre quotidien, du parcours de leur famille ou à l'aide des expériences de leur entourage. Essed a développé sa théorie en mettant au grand jour ces lectures de la réalité, ce qui a eu pour effet de les légitimer. En ce qui a trait aux membres de la culture majoritaire, ils ne peuvent nier ni les institutions qui ont été mises en place, ni l'idéologie et la socialisation qui s'y rattachent et les légitiment; ces acteurs peuvent 
ainsi poser des gestes d'exclusion consciemment ou par habitude, entre autres, dans le but d'influencer les personnes minoritaires ou même, pour contrer leur opposition.

Cette auteure identifie les manifestations d'exclusion selon trois catégories :

1) la marginalisation des personnes exclues;

2) la problématisation ou le dénigrement et l'invalidation de ces dernières;

3) le contrôle exercé sur elles.

La marginalisation découle du maintien du statu quo au sein des normes et des valeurs du groupe majoritaire; celui-ci est établi comme le cadre de référence. Des zones d'activités deviendront alors des secteurs presque protégés, par conséquent des paliers difficiles d'accès pour les personnes minoritaires. La problématisation ou le dénigrement des personnes exclues contribue à cette marginalisation et la justifie. C'est ainsi que leurs qualifications se verront sous-estimées, mais surtout leurs perceptions de la réalité et leurs expériences culturelles invalidées. Finalement, divers mécanismes de contrôle existent pour encadrer, voire contrer,l'opposition des personnes exclues. Des membres de la culture dominante pourront exercer de l'isolement culturel, de la pression pour l'assimilation, de l'humiliation, de l'intimidation et parfois du harcèlement. De surcroît, les membres de la majorité en arrivent souvent à nier l'existence même de ce processus d'exclusion.

Essed finit par en conclure que "l'attribution de la responsabilité en ce qui concerne les acteurs au sein de la majorité vise non seulement les actions entreprises, mais aussi leur inaction $[\ldots]$, leur détachement vis-à-vis des enjeux liés au racisme $[. .$. spécialement leur tolérance passive » (Essed, 2002, p. 183 [notre traduction]).

\section{La socialisation de genre appliquée aux enjeux interculturels}

Lorsqu'appliqué aux études interculturelles, il importe de préciser dès le départ que le processus de socialisation de genre va dans la veine des rôles que les hommes et les femmes adoptent à partir 
"...la socialisation différenciée selon le genre appliquée au contexte d'immigration donne l'occasion aux garçons d'avoir plus de liberté, tandis que les parents sont plus stricts avec leurs filles. » des normes qui construisent leurs milieux (Best et Thomas, 2004; Sigal et Nally, 2004). Best et Thomas (2004) avancent que les pays urbanisés aux économies plus développées et au taux de scolarisation plus élevé, où les femmes vont davantage à l'université et poursuivent une carrière professionnelle, défendent des positions plus égalitaires en ce qui trait aux hommes et aux femmes ainsi que des conceptions fondées sur les droits humains. Les femmes détiennent alors plus de contrôle par rapport à leur propre vie. Cependant, ce positionnement idéologique ne va pas jusqu'à éliminer le point de vue selon lequel les hommes seraient plus forts et plus actifs que les femmes.

En contrepartie, Sigal et Nally (2004) affirment qu'une société où l'idéologie traditionnelle légitime le contrôle des hommes sur les femmes fonctionne grâce à des normes souvent informelles qui régularisent les conduites des uns et des autres et réprimandent ceux et celles qui s'en distancient. Dans ces cultures, la famille, dont celle élargie, occupe une grande importance; le bien collectif prévaut sur les besoins individuels. Ses membres ont la responsabilité d'en assurer la continuité et la réputation.

Dans cette foulée, la socialisation différenciée selon le genre appliquée au contexte d'immigration donne l'occasion aux garçons d'avoir plus de liberté, tandis que les parents sont plus stricts avec leurs filles (Dion et Dion, 2004; Sigal et Nally, 2004; Anisef et Murphy Kilbride, 2003). Coles (2006) insiste sur la soumission attendue des jeunes filles à ce contrôle exercé sur leur vie. Dans le cadre de ce double standard, les jeunes femmes peuvent avoir des couvre-feux, des restrictions vestimentaires, des limites dans leurs sorties avec les garçons, des tâches domestiques accrues ou des responsabilités à l'égard de la fratrie plus jeune (Soàrez-Orozco et Soàrez-Orozco, 2001). Pour Dion et Dion (2001; 2004), elles deviennent alors les futures garantes des valeurs familiales et celles auxquelles on se fie pour perpétuer la culture d'origine. C'est ainsi que les jeunes femmes de parents immigrants sont plus sujettes à vivre des conflits avec leur entourage en raison des rôles traditionnels prescrits selon le genre et imbriqués dans leur culture d'origine (Dion et Dion, 2001; 2004; Sigal et Nally, 2004; Anisef et Murphy Kilbride, 2003; Soàrez-Orozco et Soàrez-Orozco, 2001). 
Dans la lignée de cette recension des écrits en deux volets qui touche à la fois l'exclusion au quotidien (Essed) et la socialisation différenciée de genre, quelles sont les manifestations d'exclusion sociale qu'affrontent des jeunes femmes d'origine somalienne, chinoise et libanaise, ainsi que leurs communautés? Quelles stratégies de résistance ou d'adaptation adoptent-elles pour y faire face?

\section{Une méthodologie proche du discours des acteurs}

"Nous visons ainsi

à saisir la réalité de jeunes femmes issues de ces trois communautés qui different au chapitre des expériences d'exclusion et des étapes migratoires, un aspect encore peu exploré."
Pour répondre à ces questions, une analyse secondaire exploratoire a été réalisée à partir des données recueillies sous le thème Family Circumstances and Gender Issues dans le cadre de la recherche Communities Within : Diversity and Exclusion in Ottawa (Moscovitch et Mohamoud, 2005; CPSO, 2008) menée par le Conseil de planification sociale d'Ottawa de 2005 à 2007. Cette étude du CPSO visait au départ à saisir les enjeux particuliers d'exclusion et d'inclusion au sein de familles d'origine somalienne, chinoise et libanaise, de même qu'à sonder si ces processus étaient vécus différemment en fonction du genre de ses membres. La grille d'entrevue touchait ces trois aspects : 1) les sentiments d'identité, d'appartenance et d'exclusion sociale; 2) les enjeux intergénérationnels vécus sur trois générations; 3) les enjeux particuliers au genre. La présente analyse secondaire (Turgeon et Bernatchez, 2003) reconsidère ces données, cette fois-ci à la lumière des écrits d'Essed sur l'exclusion vécue au quotidien et de ceux portant sur les enjeux de socialisation différenciée selon le genre. Nous visons ainsi à saisir la réalité de jeunes femmes issues de ces trois communautés qui different au chapitre des expériences d'exclusion et des étapes migratoires, un aspect encore peu exploré.

Au cours de l'hiver 2006, le CPSO a recueilli des données d'entrevue auprès de douze groupes d'entretien — quatre groupes pour chacune des trois communautés - lesquels étaient ainsi répartis : un de jeunes femmes, un de jeunes hommes, un de mères et un de pères. Par le biais de la radio ethnoculturelle ou dans des 
lieux de regroupement propres à chacun des groupes, telles les communautés virtuelles, les églises ou les mosquées, 64 personnes ont été recrutées dans divers milieux, centres culturels, collèges, universités ou centres communautaires. Des efforts notables ont été déployés pour joindre des francophones des communautés somalienne et libanaise. Lors du recrutement, cette recherche leur a été présentée comme une occasion privilégiée de réfléchir sur leur communauté et de donner une voix à leur quotidien, ce qui contribuerait à fournir des pistes d'action aux décideurs politiques.

Cet échantillon en est un de volontaires regroupés selon la méthode boule de neige et il est typique (Mayer et Deslauriers, 2000) en ce sens que les participants devaient remplir certains critères ou présenter des caractéristiques propres à leur communauté (âge, rôle dans la famille, voisinage, langue officielle canadienne utilisée, temps passé au Canada, appartenance à une première ou à une deuxième génération de personnes immigrantes).

La communauté somalienne est représentée par 21 participants qui demeurent au Canada depuis en moyenne 15 ans, durée qui concorde avec l'arrivée massive au pays, et spécialement à Ottawa, de personnes réfugiées de Somalie. Les deux groupes de jeunes réunissent des adolescents et des adolescentes de 16 à 18 ans qui vont tous à l'école, soit au secondaire ou à l'université. Les parents ont en moyenne 40 ans et un degré universitaire. Aucune question directe n'a été posée aux répondants quant à leur revenu, car il s'agit d'un sujet délicat. On a plutôt demandé aux participants de se classer sur une échelle de revenu. Leurs réponses correspondaient aux données suivantes :le revenu médian de la communauté somalienne est de 11693 \$, soit $39 \%$ de celui d'Ottawa qui s'élève à $30226 \$$; plus de $55 \%$ de ses membres vivent avec un revenu familial de $20000 \$$ ou moins (Mohamoud et Mulenga, 2006).

De même, 21 participants représentent la communauté chinoise, la deuxième minorité visible en importance à Ottawa. Ils sont tous d'immigration récente et ont en général une formation universitaire, ce qui est conforme aux statistiques canadiennes entourant cette communauté (Statistique Canada, 2007c). Le 
groupe des jeunes en compte certains qui, âgés de 20 à 26 ans, sont venus seuls pour faire des études universitaires. Le groupe des parents touche une clientèle assez diversifiée, âgée de 30 à 50 ans. Des trois groupes ethnoculturels visés dans cette étude, la communauté chinoise présente la situation économique la plus avantageuse. À ce titre, le revenu médian des membres de ce groupe est de 23157 \$, soit $77 \%$ de celui de la population d'Ottawa. Par contre, $41 \%$ d'entre eux ont un revenu de $20000 \$$ ou moins (Mohamoud et Mulenga, 2006).

De son côté, la communauté libanaise est représentée par 22 participants, lesquels vivent au Canada depuis longtemps et sont pour la plupart d'ascendance chrétienne. Le fait de n'avoir pu joindre davantage de personnes d'immigration récente ou d'ascendance musulmane constitue une limite à cette étude. Les chefs de file de cette communauté l'expliquent à partir de la discrimination que ces personnes subissent depuis les événements du 11 septembre 2001 (CPSO, 2008). Les jeunes participants aux groupes d'entretien sont de deuxième génération ou sont arrivés lorsqu'ils étaient enfants. Ils sont âgés aujourd'hui de 18 à 24 ans. Les parents, âgés de 35 à 60 ans, vivent au Canada depuis au moins 15 ans, certains depuis une trentaine d'années. Leur formation est variée, allant du diplôme d'études secondaires à la maîtrise. Plusieurs travaillent dans des services ou en sont propriétaires; la moitié d'entre eux gagnent 17602 \$ par année, soit $58 \%$ du revenu médian d'Ottawa (Mohamoud et Mulenga, 2006).

S'étendant en moyenne sur deux heures, les entrevues de groupe se sont déroulées en anglais ou parfois, avec l'aide d'un interprète, dans la langue d'origine des participants - cette section du verbatim ayant subséquemment été traduite en anglais. Les francophones n'ont éprouvé aucune difficulté à participer à des entrevues tenues en anglais. Les entretiens ont été menés par un des membres de l'équipe de recherche du même genre que les participants de ce groupe et par un membre de la communauté d'origine, aussi du même genre que les participants.

Les verbatim ont été triés à l'aide du programme N-Vivo 7 , codifiés à partir d'une analyse de contenu (Huberman et Miles, 1991). Pour notre analyse secondaire, les résultats ont été analysés 
"Cette étape a permis de constater des consensus et d'élaborer des scénarios d'exclusion pour chaque communauté. Puis, la comparaison de ces scénarios a permis de dégager une cohérence conceptuelle. » de manière déductive selon les trois grandes catégories qu'Essed associe à l'exclusion vécue au quotidien, soit la marginalisation, la problématisation et le contrôle. De plus, ils ont été analysés de manière inductive à partir de catégories construites en fonction de la socialisation de genre. Ces deux angles permettront de présenter un portrait plus global des rapports que ces jeunes femmes ont avec leur environnement. Ces résultats ont surtout été analysés de façon verticale, selon la méthode d'étude de cas, afin de suivre l'angle d'analyse du CPSO. Des constats transversaux ont aussi été dégagés.

En suivant les stratégies d'interprétation de données élaborées par Huberman et Miles (1991), les énoncés ont tout d'abord été comptés à l'intérieur des entretiens issus de chacune des communautés, en portant une attention toute particulière aux propos des jeunes femmes. Cette étape a permis de constater des consensus et d'élaborer des scénarios d'exclusion pour chaque communauté. Puis, la comparaison de ces scénarios a permis de dégager une cohérence conceptuelle. Un processus de validation interjuges a été intégré (Huberman et Miles, 1991); ces juges étant d'origines disciplinaires et ethnoculturelles différentes, la qualité de ce processus s'en est trouvée accrue.

Du fait qu'il s'agit d'une analyse secondaire de données issues d'études de cas, on peut noter des expériences hétérogènes vis-àvis quelques aspects des résultats; cela peut constituer une limite, compensée toutefois par la richesse de l'induction qui s'en dégage. Comme d'autres recherches qualitatives, les résultats peuvent être théoriquement généralisés (Pires, 1997) à l'ensemble des membres de ces communautés. Même si la saturation des données recueillies a été atteinte, la généralisation empirique (Pires, 1997) doit être faite avec précaution, étant donné le petit nombre de participants. Il est difficile de prévoir les dynamiques propres à d'autres contextes culturels. 


\section{Des jeunes femmes et leur communauté : des actrices}

Pour mieux refléter les expériences d'exclusion de même que les étapes migratoires qui different d'une communauté à l'autre, nous présentons séparément les résultats relatifs à chacune d'elles. Les propos des jeunes femmes seront tout d'abord contextualisés à l'aide de réflexions sur l'exclusion sociale que doit subir l'ensemble de leur communauté. Ainsi, les propos recueillis dans l'ensemble des groupes d'une même communauté servent à dresser un portrait plus global de l'exclusion que les jeunes femmes vivent au quotidien. Nous dégagerons aussi quelques stratégies d'adaptation ou de résistance qu'elles adoptent pour pallier un tel vécu.

\section{La communauté somalienne et la révolte de jeunes femmes}

Tous les membres de la communauté somalienne interrogés dans le cadre de ces entretiens rejettent l'expression "minorité visible " qui, selon eux, les marginalise en mettant l'accent sur ce qu'ils ne sont pas, et non sur ce qu'ils sont et ce qu'ils ont. En corollaire, cette expression établit comme étant la norme le statut des personnes caucasiennes. À leurs yeux, l'expression «minorité

"C'est ainsi que cette assise à toutes leurs réflexions sur l'exclusion sociale rejoint d'emblée ce qu'Essed identifie comme de l'eurocentrisme, la base même de la marginalisation et de l'ensemble des manifestations d'exclusion ethnoculturelle." visible " constitue un rappel constant d'une position sociale inférieure de personnes par rapport à d'autres, dites "invisibles". Comme le précise ce père de famille, " ce terme me remémore comment je suis exclu de certains bénéfices auxquels d'autres ont accès ». C'est ainsi que cette assise à toutes leurs réflexions sur l'exclusion sociale rejoint d'emblée ce qu'Essed identifie comme de l'eurocentrisme, la base même de la marginalisation et de l'ensemble des manifestations d'exclusion ethnoculturelle. Un père résume très bien cette distinction et discute de son impact sur les enfants et les jeunes:

"Je viens d'une culture où j'étais la norme. Mon identité est donc intacte. Je ne pense pas qu'un étiquetage va me décrire, donc je le rejette quand je l'entends. D'un autre côté, je suis inquiet pour 
"Quant aux jeunes femmes d'origine somalienne, elles ont à surmonter au quotidien nombre de limitations. » mes enfants. Parce qu'ils grandissent dans cette société, j'ai peur qu'ils intériorisent l'aspect négatif qui vient avec ce terme; je suis inquiet que cela va les limiter.»

Il importe de répéter que le revenu médian de la communauté somalienne est de 11693 \$, à peine le tiers de celui des résidents d'Ottawa (30 226 \$), et ce, même après 15 ans de résidence au Canada. Le statut de réfugié non parrainé a retardé l'intégration sociale et économique des Somaliens. En effet, pour avoir un emploi, il faut avoir le statut de résident permanent ou un permis de travail; les deux sont difficiles à obtenir pour un réfugié. L'arrivée en grand nombre de ces réfugiés individuels, sans encadrement ou soutien d'organismes humanitaires, a provoqué des discours négatifs dans les médias et engendré des préjugés dans les voisinages, les écoles, les autobus, etc.

Quant aux jeunes femmes d'origine somalienne, elles ont à surmonter au quotidien nombre de limitations. Elles rencontrent des restrictions quand les normes liées à leur religion musulmane ne correspondent pas aux façons de faire de la majorité, par exemple, des gymnases ou des périodes de natation pour jeunes filles, des fêtes sans alcool, la tenue vestimentaire. Une répondante illustre cette forme d'exclusion et mentionne des stratégies qu'adoptent certaines jeunes filles, et même des employeurs, afin de s'y adapter :

«Ma jeune sœur a de la difficulté à se trouver un emploi parce qu'elle met des jupes. Beaucoup des emplois qui demandent des uniformes, comme chez Tim Hortons, sont inaccessibles aux femmes musulmanes. Dans d'autres milieux, elles mettent des jupes de la couleur et du même matériel que des pantalons."

Ces jeunes femmes se voient également marginalisées parce que certains employeurs, en se fondant sur des stéréotypes, leur réservent des tâches exigeant moins d'habiletés. Il en sera de même pour des enseignants qui ne voient pas leurs talents, réduisent leurs attentes, les disqualifient, dénigrent leurs capacités : 
"...qu'un humour raciste ne soit pas réprimandé par une autorité, tout cela peut réduire une personne au silence... "
«Je fais partie d'une classe de douance en sciences. Après deux semaines, l'enseignante m'a dit :"Ce n'est pas un jeu ici, il faut travailler”. Après le $1^{\text {er }}$ test, elle a vu que j'étais capable de faire le travail. J'étais la seule Somalienne quand l'école en a plusieurs. Nous avions un projet de groupe. J'ai fait presque tout le travail. Quand elle nous a félicités, elle ne m'a même pas mentionnée. Les autres élèves ont dit :"Maymuun était là aussi". L'enseignante a dit : "Vous avez probablement fait la majorité du travail". Je la hais.»

Dans la lignée d'Essed, on constate qu'ainsi marginalisée, cette jeune fille voit ses efforts invalidés : on lui attribue une incompétence dès le départ, et même après de premiers succès. De plus, qu'un geste d'exclusion ne soit pas reconnu, que des paroles humiliantes ne soient pas condamnées, qu'un humour raciste ne soit pas réprimandé par une autorité, tout cela peut réduire une personne au silence, comme en témoigne l'exemple suivant. Il illustre ce qu'Essed postule lorsqu'elle soutient que les mesures de contrôle mises en place permettent la perpétuation des gestes d'exclusion et que le déni et l'inaction constituent une forme courante de mesures de contrôle :

"J'ai apporté le MP3 de mon frère au travail. Une de mes collègues m'a dit : "C'est à toi?" Mon superviseur lui a répondu :"Elle est Somalienne. C'est certain que ce n'est pas à elle. Penses-tu que les Somaliens ont assez d'argent pour s'en acheter?" J'ai averti l'autre superviseur et elle m'a dit que je n'aurais pas dû l'apporter. Ce milieu de travail était vraiment raciste. [...]. Deux de mes collègues ne me parlaient pas. Elles se parlaient entre elles et émettaient des commentaires racistes: "Nous ne nous tenons pas avec des musulmans!" "

En plus de leurs contacts parfois difficiles avec des membres ou des institutions de la majorité, ces jeunes femmes vivent des défis 
"...ces jeunes femmes vivent des défis à l'intérieur de leur famille où, selon leurs dires, elles deviennent de secondes mères du fait que leur mère travaille pour assurer leur bien-être. » à l'intérieur de leur famille où, selon leurs dires, elles deviennent de secondes mères du fait que leur mère travaille pour assurer leur bien-être. De lourdes responsabilités domestiques s'ajoutent à leurs devoirs scolaires. Cette surcharge apparait aussi comme un obstacle à leur intégration sociale. De surcroît, certaines subissent des restrictions quant à leurs allées et venues. Ces contraintes sont souvent rattachées aux tâches ménagères : au rôle inhérent des femmes et à l'obéissance attendue de la part des filles. Dans d'autres contextes, Soàres-Orozco et Soàrez-Orozco (2001) notent que de telles limitations ont un effet protecteur sur les jeunes filles immigrantes qui présentent moins de conduites antisociales que les garçons. Cet effet protecteur englobe les contacts plus étroits avec les parents et la famille élargie, une meilleure supervision et des loisirs socialement intégrés. En ce qui concerne nos répondantes, elles se plaignent régulièrement à leurs parents, tentent de résister, réclament plus de la liberté, tout cela en vain; chaque fois, elles sont réduites à cet état d'invalidation.

\section{La communauté chinoise et le désir d'assimilation de jeunes femmes}

À l'instar des participants d'origine somalienne, les répondants de la communauté chinoise rejettent d'emblée l'expression " minorité visible ». Ils s'inquiètent eux aussi pour l'intégration sociale de leurs enfants comme le décrit si bien ce père d'origine chinoise :

"Le statut de se faire appeler "minorité visible" a des bons et des mauvais côtés. Il y a au moins des programmes pour les minorités visibles. Par contre, la perception des autres à l'égard des vismins n'est pas bonne. Nous recevons souvent un traitement discriminatoire. Par exemple, on fait une petite erreur à l'ouvrage, elle est vue comme énorme par les autres. Encore plus, nos enfants ne s'intègrent pas facilement à l'école. » 
Pour mieux saisir l'ampleur de l'exclusion vécue par la communauté chinoise, il faut souligner que $70 \%$ des immigrants récents, étape migratoire des participants à cette étude, ont un diplôme d'études postsecondaires. Or, malgré ce haut niveau d'éducation, leur taux de chômage est de 8,6 \%, soit le double de celui des résidents d'Ottawa qui ne sont pas de minorités visibles. Au moment de la collecte de données, le taux de chômage canadien se situait à $5 \%$. Le manque de réseaux d'accès à l'emploi limite leurs possibilités d'intégration sociale et économique : «S'il faut un réseau pour avoir un emploi, comment peut-on affirmer que nous sommes dans une société équitable? » De plus, parler l'anglais avec un accent et ne pas avoir d'expérience canadienne de travail sont d'autres barrières importantes à leur employabilité.

"Devant les difficultés d'intégration que connaît leur entourage, les jeunes femmes rencontrées vont tout d'abord exprimer une ferme volonté de s'intégrer à leur milieu. Préoccupées par le fait d'enrichir leurs relations et de bâtir des amitiés, elles prendront des moyens concrets pour s'implanter..."
Devant les difficultés d'intégration que connaît leur entourage, les jeunes femmes rencontrées vont tout d'abord exprimer une ferme volonté de s'intégrer à leur milieu. Préoccupées par le fait d'enrichir leurs relations et de bâtir des amitiés, elles prendront des moyens concrets pour s'implanter :

"J'ai commencé à aller à l'église pour avoir des amis. Mon but était simple : rencontrer des personnes et améliorer mon anglais. Cependant, leur but est que tu fasses partie du groupe. La religion est vraiment pour rencontrer des personnes : tu n'as pas vraiment besoin de croire à tout, à $100 \%$.»

Face à diverses manifestations d'exclusion, ces jeunes femmes vont déployer de nombreux efforts pour s'adapter et s'intégrer à la culture majoritaire. Par exemple, elles vont changer leur prénom ou participer à des activités propres à la culture dominante : "Noël est un tel festival canadien ici. C'est une grande chance pour connaître quelque chose de neuf et se faire des amis. " Elles modifient leur apparence physique dans le but de ressembler le plus possible à des Caucasiennes. C'est ainsi que l'une d'elles met de la couleur dans ses cheveux, une autre n'achète que des vêtements griffés. Une autre rêve d'une peau blanche; ses amies appartenant à la majorité canadienne lui disent, et elle en est très fière : « Ta 
"Outre un sentiment d'échec face à tous les efforts déployés, cette expérience peut se transformer chez elles en un obstacle supplémentaire d'intégration sociale, cette fois-ci dans leur propre communauté." peau est parfaite, tu n'as pas à t'inquiéter. Tu as l'air tellement en santé avec ta peau couleur de chocolat au lait".

Or, le fait qu'elles valorisent des aspects physiques qui sont différents des leurs, voire qui leur sont opposés, peut les amener à se percevoir comme non-Caucasiennes (Zhou et Lee, 2004). Outre un sentiment d'échec face à tous les efforts déployés, cette expérience peut se transformer chez elles en un obstacle supplémentaire d'intégration sociale, cette fois-ci dans leur propre communauté. Inman (2006) suggère que de telles jeunes femmes, encore plus celles de deuxième génération, sont éventuellement perçues par leur milieu d'origine comme étant trop intégrées à la société majoritaire. Elles perdent alors de la crédibilité à l'intérieur du milieu même où elles sont nées et vivent en conséquence une double invalidation.

\section{La communauté libanaise et la double vie de jeunes femmes}

"Ils peuvent d'ailleurs aisément se faire passer pour des personnes d'origine européenne, ayant moins de différences raciales que les membres des deux groupes précédents. "
Comme les participants des deux communautés précédentes, ceux de la communauté libanaise rejettent l'expression «minorité visible ". De surcroît, ils affirment un profond sentiment d'appartenance à l'identité canadienne : «Nous ne sommes pas des minoritaires. Nous ne nous sentons pas ainsi. Nous sommes des citoyens canadiens et recevons les mêmes bénéfices que les autres Canadiens. Nous faisons tout ce que nous pouvons pour notre pays, le Canada."

Outre le fait que les répondants y demeurent depuis longtemps (certains depuis trente ans; quelques jeunes sont de deuxième génération), ils se sentent mieux intégrés que les autres communautés. Ils peuvent d'ailleurs aisément se faire passer pour des personnes d'origine européenne, ayant moins de différences raciales que les membres des deux groupes précédents. Dans le cadre quotidien, ils vont adopter de nombreuses stratégies pour minimiser toute différence évidente avec la majorité, soit adopter des noms sans connotation arabe ou des tenues vestimentaires à la mode. La majorité des participants de cette étude étant de descendance chrétienne, ils ont porté des croix 
«Or, les personnes nées à l'étranger doivent faire face à de longs délais pour atteindre le niveau maximal de sécurité nécessaire à certains emplois; parfois, les niveaux de sécurité sont même accrus afin d'exclure certains types de personnes ou pour en favoriser d'autres. " ou d'autres marqueurs religieux dans la foulée des événements du 11 septembre 2001 pour bien se protéger des stéréotypes dirigés contre les personnes libanaises d'ascendance musulmane et continuer d'être intégrés à la majorité. Un jeune homme affirme : "Je n'ai jamais vécu de discrimination. Il y a toujours eu une solide évidence d'intégration".

Mais en dépit de cette intégration sociale et de ce sentiment d'appartenance, des obstacles importants limitent l'intégration économique des membres de cette communauté. Une de ces barrières structurelles s'avère la vérification des antécédents et l'émission des certificats de sécurité. Or, les personnes nées à l'étranger doivent faire face à de longs délais pour atteindre le niveau maximal de sécurité nécessaire à certains emplois; parfois, les niveaux de sécurité sont même accrus afin d'exclure certains types de personnes ou pour en favoriser d'autres. À ce chapitre, les événements du 11 septembre 2001 ont marqué les membres de la communauté libanaise de façon particulière. Pour $18 \%$ parmi eux, comparativement à $8,6 \%$ pour les autres minorités visibles d'Ottawa et à 10,2\% pour la population caucasienne, la réponse a été de créer des petites entreprises familiales de services, préférant selon leurs dires " être leur propre roi ».

Même si les jeunes femmes d'origine libanaise interviewées sont intégrées socialement et économiquement, elles perçoivent quotidiennement de subtiles différences de réaction chez certains employeurs, enseignants ou collègues, par rapport auxquels elles se sentent désavantagées. Les diverses restrictions venant de leur famille provoquent de plus chez elles un grand sentiment d'injustice. Plusieurs jeunes filles se plaignent que les garçons autour d'elles peuvent sortir tard le soir et amener des amis à la maison, alors que leur sont interdits les appels venant de garçons, les sorties passé une certaine heure, les couchers chez une copine ou tout simplement les sorties entre amies. L'étude de McIrvin Abu-Laban et collab. (1999) confirme ces dires : près de $70 \%$ des jeunes canadiennes d'origine arabe rapportent des limites semblables imposées par leurs parents. 
" ...il y a un

consensus entre les

quatre groupes de cette

collectivité : l'entourage

règlemente les rapports

entre les garçons et

les filles, et ce, au

détriment de la liberté

des femmes. "
De plus, il y a un consensus entre les quatre groupes de cette collectivité : l'entourage règlemente les rapports entre les garçons et les filles, et ce, au détriment de la liberté des femmes. Cet aspect de la culture a déjà été soulevé dans deux autres études, Cyrenne (2007) et Abdulnour (2002). Ce respect des traditions va largement au-delà des pratiques actuelles au Liban. Selon les parents, la représentation dans les médias des relations entre les hommes et les femmes de la majorité soulève les craintes et justifie un tel contrôle. La désapprobation du milieu se traduit, entre autres, par les commentaires des membres des institutions religieuses - ici chrétiennes - qui surveillent les relations entre les jeunes et qui partagent avec les garçons leurs opinions sur les jeunes femmes dont ces derniers s'éprennent. Des jeunes hommes interviewés confient que de leurs amis consultent les autorités religieuses au sujet des jeunes femmes qui les intéressent. Des femmes s'en trouvent ainsi étiquetées. Certains frères jouent même le rôle de gardiens de leurs sœurs et veillent sur leur réputation :

"La réputation pour une jeune femme libanaise, c'est quelque chose de très important et si quelqu'un parle en mal d'elle ou la voit avec un garçon, cela affecte sa réputation. Il y a des parents qui mettent de la pression en disant : "Je veux savoir et être certain qui courtise ma fille et qu'il soit surveillé." Il y a donc des frères qui empêchent leurs sœurs de sortir. Ils ne veulent pas les voir dans les clubs ou parler avec des garçons. C'est très important dans la communauté libanaise. Si les filles veulent vraiment communiquer avec un homme et sortir, elles le font; mais elles le font derrière le dos des gens."

Ce témoignage jette un éclairage sur les réactions des jeunes filles à tant de contrôle. Celles rencontrées avouent mentir aux autres. Anisef et Murphy Kilbride (2003) affirment qu'elles résistent à ce contrôle social en développant une double vie. Elles disent à leur famille ce qu'elle veut entendre; elles font de même avec leurs amies de la majorité parce qu'elles ne veulent 
pas les perdre. Elles évitent ainsi une double invalidation, celle de leur communauté d'origine et celle leurs amies de la culture majoritaire.

\section{Conclusion : Des pistes de réflexion pour contrer cette double invalidation}

"Les jeunes femmes des trois communautés rencontrées font clairement face à une double invalidation, l'une venant de la culture majoritaire et l'autre de leur milieu d'origine. Cette dernière dépend de l'expérience de leur famille dans le contexte canadien et des stratégies qu'elle développe pour s'y adapter. "
Cet article avait pour but de cerner les manifestations d'exclusion sociale qu'affrontent spécialement des jeunes femmes d'origine somalienne, chinoise et libanaise, de même que les stratégies qu'elles adoptent pour s'y adapter ou y résister. Essed définit l'exclusion ethnoculturelle comme un phénomène systématique de gestes familiers et répétitifs, intégrés aux routines de la vie quotidienne. Cette auteure en identifie trois grandes catégories de manifestation :

1) la marginalisation des personnes exclues;

2) la problématisation, c.-à-d., leur dénigrement ou leur invalidation;

3) le contrôle exercé sur elles.

Les jeunes femmes des trois communautés rencontrées font clairement face à une double invalidation, l'une venant de la culture majoritaire et l'autre de leur milieu d'origine. Cette dernière dépend de l'expérience de leur famille dans le contexte canadien et des stratégies qu'elle développe pour s'y adapter. Les jeunes femmes d'origine somalienne affrontent d'importants préjugés de la part de la culture dominante, des stéréotypes dénigrants fondés sur une disqualification de leur milieu d'origine. Elles se voient aussi exclues de cette société d'accueil en raison des lourdes responsabilités domestiques qu'elles assument pour contribuer à la stabilité et à la survie économique de leur famille, leur mère devant travailler. Leurs doléances n'étant pas reçues dans leur milieu, elles se voient ainsi doublement invalidées. Leurs demandes sont jugées secondaires par leur communauté qui est confrontée à une grande exclusion économique et lutte pour sa survie. 
Les préoccupations économiques et sociales des communautés d'origine de ces jeunes femmes l'emportent sur leurs considérations personnelles : elles se voient réduites au silence. Même si elles résistent un certain temps, elles peuvent à la longue s'épuiser et perdre pieds. Ces invalidations risquent ainsi de provoquer chez elles une intériorisation des images négatives de leur communauté, des stéréotypes des femmes et des représentations désobligeantes de leurs capacités. Cela pourrait engendrer un processus de victimisation qui entraînerait ultimement une " autoexclusion ». Elles commenceraient donc leur vie d'adulte avec des limitations supplémentaires à dépasser.
Les jeunes femmes d'origine chinoise rencontrées adoptent nombre de stratégies pour pouvoir s'assimiler à la majorité, comme changer leur apparence physique et chercher activement à bâtir des amitiés avec des pairs de la majorité. En raison des limites inhérentes à de tels changements, elles se perçoivent davantage comme non-Caucasiennes; elles peuvent être rejetées par certains pairs de leur communauté d'origine qui les jugent trop assimilées. Là encore, le contexte d'immigration récente et d'insertion économique canalise la plupart des énergies de leur entourage et, conséquemment, leurs préoccupations paraissent futiles. Ces jeunes femmes sont alors victimes d'une double invalidation.

Quant aux jeunes femmes d'origine libanaise, leur communauté demeure au Canada depuis longtemps et s'y identifie avec force. Mais elle adhère beaucoup aux traditions qui entourent les rapports entre les hommes et les femmes et qui régissent, entre autres, les allées et venues des jeunes femmes. Ce respect des traditions va largement au-delà des pratiques actuelles au Liban. Il s'explique par les craintes des parents devant une certaine représentation médiatique des relations entre les hommes et les femmes appartenant à la majorité. Ces jeunes femmes refusent cette gestion de leur quotidien, mentent pour y résister et développent une double vie. Elles évitent ainsi une double invalidation, l'une venant de leur communauté d'origine et l'autre de leurs amies de la culture majoritaire.

Les préoccupations économiques et sociales des communautés d'origine de ces jeunes femmes l'emportent sur leurs considérations personnelles : elles se voient réduites au silence. Même si elles résistent un certain temps, elles peuvent à la longue s'épuiser et perdre pieds. Ces invalidations risquent ainsi de provoquer chez elles une intériorisation des images négatives de leur communauté, des stéréotypes des femmes et des représentations désobligeantes de leurs capacités. Cela pourrait engendrer un processus de victimisation qui entraînerait ultimement une " autoexclusion ". Elles commenceraient donc leur vie d'adulte avec des limitations supplémentaires à dépasser. 
En guise d'apports aux pratiques d'intervention, ces résultats indiquent que les jeunes femmes ont une fine perception de la complexité de leur situation : elles sont partagées entre leur héritage et un avenir enraciné dans la société canadienne. Mais davantage que l'on peut le penser parfois au sein des services sociaux, elles sont conscientes des conditions de vie de leur famille et de leur communauté d'une part et, d'autre part, impliquées dans leur propre vie quotidienne. Devant leurs stratégies de résistance, qui génèrent des tensions avec leurs parents, les travailleurs sociaux offrent souvent des services aux familles selon une analyse écosystémique. Or, les parents peuvent considérer ces agirs essentiellement comme des défis à leur autorité nécessitant d'être contrôlés. Ils tiennent alors peu compte du sens que les jeunes femmes leur donnent et des besoins d'autonomie qu'elles

Essed (2007) avance que les discours venant des mouvements qui luttent contre le racisme et l'exclusion ethnoculturelle font peu mention des différences de genre, sauf à travers les groupes de femmes. Or, dans une société plus juste pour chacun, la reconnaissance des enjeux particuliers de genre devient un incontournable. Sinon, comme pour ces jeunes fermmes, l'analyse fine de certaines exclusions ressenties par un sous-groupe peut être ignorée. expriment, conduites jugées différentes, voire opposées aux attentes.

Ces résultats invitent donc les travailleurs sociaux à revoir les interventions auprès de ces familles sous l'angle de l'apport de chacun de leurs membres et à remettre la socialisation de genre au centre de leurs préoccupations. Entre autres pistes, il faudrait créer des espaces : 1) pour permettre aux jeunes femmes de s'articuler et de valider leur expérience; 2) pour identifier les sources de pressions sociales et mieux contextualiser leur quotidien;3) pour favoriser avec leurs parents un dialogue respectueux qui tient compte des craintes et des aspirations de chacune des parties; 4) pour favoriser l'établissement de nouvelles normes d'agir et de solutions appropriées.

Essed (2007) avance que les discours venant des mouvements qui luttent contre le racisme et l'exclusion ethnoculturelle font peu mention des différences de genre, sauf à travers les groupes de femmes. Or, dans une société plus juste pour chacun, la reconnaissance des enjeux particuliers de genre devient un incontournable. Sinon, comme pour ces jeunes femmes, l'analyse fine de certaines exclusions ressenties par un sous-groupe peut être ignorée. Les pratiques citoyennes de ces sous-groupes peuvent être encadrées, voire assimilées et occultées par l'opinion majoritaire qui émane d'un groupe d'intérêt, comme l'est une 
communauté. Puisqu'une voix uniforme établit une meilleure force ou un meilleur rapport de force, elle donne ainsi plus de chances à ce collectif d'avoir des résultats par rapport à certaines luttes précises, mais parfois au détriment des voix plus minoritaires alors invalidées.

Pour affirmer ses positions en faveur de la justice sociale et des droits humains, le travail social a une responsabilité de reconnaitre cette invalidation des minorités au sein de minorités et de décrier cette exclusion vécue quotidiennement. Ses pratiques doivent tenir compte du racisme structurel et idéologique. Au cours des années 1990, Malcolm Payne (1991) nous prévenait d'une tendance eurocentriste du travail social, fondée sur des droits humains à caractère plus individuel. Selon d'autres cadres de référence culturelle, l'accent peut être mis sur une vision plus collective : une équité de groupe ou un réseau familial élargi où le collectif prime les besoins individuels. La décentration en faveur de l'autre, déjà présente en intervention interculturelle, contribuera à ce que nous pensions autrement la pratique familiale, la place de chacun, le rôle des jeunes femmes et les droits humains. Le travail social se doit de devenir le porte-parole de ces minorités au sein des minorités pour qu'elles contribuent également au développement social.

La recherche du Conseil de planification sociale a reçu le soutien financier de Patrimoine Canada, de la ville d'Ottawa, de Centraide Ottawa et de l'Université Carleton.

\section{Bibliographie}

ABDULNOUR, Aline (2002). Entre deux cultures : stratégies utilisées par les jeunes Canadiennes libanaises pour faire face aux conflits intergénérationnels, Mémoire déposé à l'École de service social de l'Université d'Ottawa, dirigé par Madeleine Dubois.

ANISEF, Paul, et Kenise MURPHY KILBRIDE (2003). "Conclusion : Overview and implications of the research ", dans Paul Anisef et Kenise Murphy Kilbride (dir.), Managing two worlds : The experiences $\mathcal{E}$ concerns of immigrant youth in Ontario, Toronto, CSPI. p. 235-272.

BEST, Deborah L., et Jennifer J.THOMAS (2004). Cultural diversity and cross-cultural perspectives, dans Alice H. Eagly, Anne E. Beall et Robert J. Sternberg, The Psychology of gender, New York et London, The Guilford Press, p. 296-327. 
BOUDON, Raymond, et collab. (1993). Dictionnaire de la sociologie, Paris, Larousse.

COLES, Roberta (2006). Race and family : A structural approach, Thousand Oaks, Sage Publications.

CONSEIL DE PLANIFICATION SOCIALE D'OTTAWA (2005). Rationale for selecting the project's case study communities, Ottawa : Communities Within : Diversity and exclusion in Ottawa.

CONSEIL DE PLANIFICATION SOCIALE D'OTTAWA (2008). Exclusion within Ottawa's visible and ethnic Minority Communities: The inter-case study, Ottawa : Communities Within : Diversity and exclusion in Ottawa.

CYRENNE, Sophie (2007). Les défis dans les relations intergénérationnelles chez des familles issues de l'immigration: le cas de familles d'origine libanaise, Mémoire déposé à l'École de service social de l'Université d'Ottawa, dirigé par Marie Drolet.

CUSHING, Pamela (2003). Policy Approaches to Framing Social Inclusion and Social exclusion : An Overview, Toronto, Institut Roeher Institute.

DEHAAN, Arjan (1999). Social Exclusion : Towards a Holistic Approach of Deprivation, réf. du 4 avril 2009, http://www.dse.de/ef/poverty/dehaan.htm.

DION, Karen K., et Kenneth L. DION (2001). "Gender and cultural adaptation in immigrant families ", Journal of Social Issues, Vol. 57, No 3, p. 511-521.

DION, Karen K., et Kenneth L. DION (2004). « Gender, immigrant generation, and ethnocultural identity ", Sex Roles, Vol. 50, No 5, p. 347-355.

ESSED, Philomena (1991). Understanding everyday racism : An interdisciplinary theory, Newbury Park, London et New Delhi, Sage Publications.

ESSED, Philomena (2002). "Everyday racism : A new approach to the study of racism ", dans Philomena Essed et David T. Goldberg, Race critical theories : Text and context, Massachusetts et Oxford, Blackwell Publishers Inc., p. 176-194.

ESSED, Philomena (2007). "Multi-identifications and Transformations : Reaching beyond Racial and Ethnic Reductionisms ", dans Genevieve Johnson, et Randy Enomoto, Race, Racialization, and Antiracism In Canada and Beyond, Toronto, University of Toronto Press, p. 233-252.

GOLDBERG, David T. (2007). "Raceless States », dans Genevieve Johnson et Randy Enomoto Race, Racialization, and Antiracism in Canada and Beyond, Toronto, University of Toronto Press, p. 206-232.

GREENBER G, Lee (2008). 1 in 5 Ottawans is self-described visible minority : Non-European community growing 3 times faster than rest of population, Ottawa, Ottawa Citizen, 3 avril 2008.

HAYANI, Ibrahim (1999). "Arabs in Canada : Assimilation or integration? », dans Michael Suleiman (dir.), Arabs in America : Building a new future Philadelphia, Temple University Press, p. 285-303.

HUBERMAN, Michael A., et Matthew B. MILES (1991). «Analyse des données qualitative », Recueil de nouvelles méthodes, Bruxelles, De Boeck Université.

INMAN, Arpana G. (2006). "South Asian women : Identities and conflicts ", Cultural diversity and ethnic minority psychology, Vol. 12, No 2, p. 306-319.

MAYER, Robert, et Jean-Pierre DESLAURIERS (2000). "Quelques éléments d'analyse qualitative : L'analyse de contenu, l'analyse ancrée, l'induction analytique et le récit de vie ", dans Robert Mayer et collab. (dir.), Méthodes de recherche en intervention sociale Montréal, Gaétan Morin Éditeur, p.159-190. 
MCIRVIN ABU-LABAN, Sharon, et Baha ABU-LABAN (1999). «Teens-Between :The Public and Private Spheres of Arab-Canadian Adolescents ", dans Michael Suleiman (dir.), Arabs in America : Building a new future, Philadelphia, Temple University Press, p. 113-128.

MOHAMOUD, Hindia, et Chola MULENGA (2006). Some socio-economic characteristics of visible minority groups in Ottawa, Ottawa, The social planning council of Ottawa.

MOSCOVITCH,Allan, et Hindia MOHAMOUD (2005). Communities within : Diversity and exclusion in Ottawa, Ottawa, The Social Planning Council of Ottawa.

OFFICE DES AFFAIRES FRANCOPHONES (2005). "Fait saillants - Profil des minorités raciales francophones en Ontario ", réf. du 15 juin 2010, http://www.ofa.gov.on.ca/docs/ stats-2005minorites-fs.pdf.

OMIDVAR, Ratna, et Ted RICHMOND (2003). "Immigrant settlement and social inclusion in Canada ", Laidlaw's Foundation Working paper series on social inclusion, réf. du 15 mars 2010, http:// www.laidlawfdn.org/cms/file/children/richmond.pdf.

PAPILLON, Martin (2004). "Immigration, Diversity and Social Inclusion in Canadian Cities ", CPRN Discussion Papers, $N^{\circ}$ F27, December.

PAYNE, Malcolm (1991). Modern Social Work Theory, New York, Palgrave Macmillan Ltd.

PIRES,Alvaro (1997). «Échantillonage et recherche qualitative :Essai théorique et méthodologique », dans Jean POUPART et collab. (dir.), La recherche qualitative: Enjeux épistémologiques et méthodologiques, Montréal, Gaétan Morin Éditeur, p. 113-169.

REITZ, Jeffrey G. (1998). Warmth of the Welcome :The Social Causes of Economic Success for Immigration in Different Nations, Boulder, Colorado, Westview.

ROGERS, Dave, et Kathryn YOUNG (2007). Arabic speakers change the face of Ottawa : After English and French, Arabic is the most common language in the region, Ottawa, Ottawa Citizen, 5 décembre 2007.

SALOOJEE, Anver (2003). Social inclusion, anti-racism and democratic citizenship, Toronto, Laidlaw Foundation Working Paper Series- Perspectives on Social Inclusion.

SEN, Amartya (2000). Social Exclusion : Concept, Application and Scrutiny, Manila, Asian Development Bank.

SIGAL, Janet, et Maureen NALLY (2004). "Cultural perspectives on gender ", dans Michele A. Paludi, Praeger guide to the psychology of gender, London, Praeger, p. 27-40.

STATISTIQUES CANADA (2007a). Portrait de la population Canadienne en 2006 : Résultats, réf. du 15 juin 2007, http://www12.statcan.ca/français/census06/analysis/popdwell/index.cfm.

STATISTIQUES CANADA (2007 b). Population selon le statut d'immigrant et la période d'immigration, chiffres de 2006, pour le Canada, les provinces et les territoires - Données - Échantillon (20\%), réf. du $1^{\text {er }}$ avril 2008, http://www12.statcan.ca/français/census06/data/Highlights/Immigration/ About.cfm

STATISTIQUES CANADA (2007c). La communauté chinoise au Canada : une communauté grandissante, réf. du 15 juin 2007, http://www.statcan.ca/français/freepub/89-621-XIF/89-621-XIF2006001. htm.

STATISTIQUES CANADA (2008). La mosaïque ethnoculturelle du Canada, recensement de 2006, réf. du 2 avril 2008, http://www12.statcan.ca/français. 
SUÀREZ-OROZCO, Carola, et Marcelo M. SUÀREZ-OROZCO (2001). "The psychosocial experience of immigration ", Children of Immigration, Cambridge, Massachussets et London, Harvard University Press, p. 67-121.

TURGEON, Jean, et Jean BERNATCHEZ (2003). "Chapitre 17 : Les données secondaires », dans Benoît GAUTHIER (dir.), Recherche Sociale : De la problématique à la collecte des données, Québec, Presses de l’Université du Québec, p. 431-468.

ZHOU, Min, et Jennifer LEE (2004). «Introduction :The making of culture, identity and ethnicity among Asian American youth ", Asian American Youth : Culture, identity and ethnicity, New York et London, Routledge, p. 1-30. 